OPEN ACCESS

Edited by:

Jack Tsao

The University of Tennessee,

Knoxville, United States

Reviewed by:

Audrey Zucker-Levin,

University of Saskatchewan, Canada

Jun Yao,

Northwestern University,

United States

Briana Nicole Perry,

Boston University, United States

*Correspondence:

Oskar C. Aszmann

oskar.aszmann@meduniwien.ac.at

${ }^{\dagger}$ These authors have contributed

equally to this work

Specialty section:

This article was submitted to

Neuroprosthetics,

a section of the journal

Frontiers in Neuroscience

Received: 24 October 2017 Accepted: 19 November 2018

Published: 04 December 2018

Citation:

Sturma A, Hruby LA, Prahm C, Mayer JA and Aszmann OC (2018) Rehabilitation of Upper Extremity Nerve Injuries Using Surface EMG

Biofeedback: Protocols for Clinical Application. Front. Neurosci. 12:906. doi: 10.3389/fnins.2018.00906

\section{Rehabilitation of Upper Extremity Nerve Injuries Using Surface EMG Biofeedback: Protocols for Clinical Application}

\author{
Agnes Sturma ${ }^{1,2,37}$, Laura A. Hruby ${ }^{1+}$, Cosima Prahm¹, Johannes A. Mayer ${ }^{1}$ and \\ Oskar C. Aszmann ${ }^{1,4 *}$
}

' Christian Doppler Laboratory for Restoration of Extremity Function, Department of Surgery, Medical University of Vienna, Vienna, Austria, ${ }^{2}$ Health Assisting Engineering, University of Applied Sciences FH Campus Wien, Vienna, Austria, ${ }^{3}$ Neuromechanics and Rehabilitation Technology Group, Department of Bioengineering, Imperial College London, London, United Kingdom, ${ }^{4}$ Division of Plastic and Reconstructive Surgery, Department of Surgery, Medical University of Vienna, Vienna, Austria

Motor recovery following nerve transfer surgery depends on the successful reinnervation of the new target muscle by regenerating axons. Cortical plasticity and motor relearning also play a major role during functional recovery. Successful neuromuscular rehabilitation requires detailed afferent feedback. Surface electromyographic (sEMG) biofeedback has been widely used in the rehabilitation of stroke, however, has not been described for the rehabilitation of peripheral nerve injuries. The aim of this paper was to present structured rehabilitation protocols in two different patient groups with upper extremity nerve injuries using sEMG biofeedback. The principles of sEMG biofeedback were explained and its application in a rehabilitation setting was described. Patient group 1 included nerve injury patients who received nerve transfers to restore biological upper limb function $(n=5)$ while group 2 comprised patients where biological reconstruction was deemed impossible and hand function was restored by prosthetic hand replacement, a concept today known as bionic reconstruction $(n=6)$. The rehabilitation protocol for group 1 included guided SEMG training to facilitate initial movements, to increase awareness of the new target muscle, and later, to facilitate separation of muscular activities. In patient group 2 sEMG biofeedback helped identify EMG activity in biologically "functionless" limbs and improved separation of EMG signals upon training. Later, these SEMG signals translated into prosthetic function. Feasibility of the rehabilitation protocols for the two different patient populations was illustrated. Functional outcome measures were assessed with standardized upper extremity outcome measures [British Medical Research Council (BMRC) scale for group 1 and Action Research Arm Test (ARAT) for group 2] showing significant improvements in motor function after sEMG training. Before actual movements were possible, sEMG biofeedback could be used. Patients reported that this visualization of muscle activity helped them to stay motivated during rehabilitation and facilitated their understanding of 
the re-innervation process. SEMG biofeedback may help in the cognitively demanding process of establishing new motor patterns. After standard nerve transfers individually tailored SEMG biofeedback can facilitate early sensorimotor re-education by providing visual cues at a stage when muscle activation cannot be detected otherwise.

Keywords: nerve reconstruction, upper extremity rehabilitation, surface electromyography, neuro-rehabilitation, nerve transfer, prosthetic rehabilitation

\section{INTRODUCTION}

Biofeedback applications measure biological information and feed them back to the patient to increase awareness and control over biological processes (Neblett, 2016). With the advent of information technology, computerized multimedia displays allow highly sophisticated and detailed recordings of real-time biological data that otherwise would not be identified by both patient and clinician (Giggins et al., 2013). Representing one of the oldest biofeedback modalities sEMG provides feedback of muscle activity by conversion of myoelectrical activity into visual and/or auditory information (Cram, 2003; Giggins et al., 2013; Kim, 2017), e.g., displayed as color-coded graphs on a computer screen with the device itself in front of the patient, as shown in Figures 1, 2. While Figure 1 shows training with a stand-alone 2-channel device with dry electrodes (MyoBoy ${ }^{\circledR}$ by Ottobock Healthcare, Duderstadt, Germany), Figure 2 includes a set-up with wet electrodes and device software used to display muscular activity (TeleMyo $2400 \mathrm{~T}^{\mathrm{G}}{ }^{\circledR}$ by Noraxon, United States). As illustrated in these figures wet electrodes have a thin coating of conductive gel on their surface, which supports electrical conductivity and makes them self-adhesive, but also allows single-use only. In contrast to that dry electrodes do not use any gel and need to be attached to the skin (e.g., with tape).

Nerve injuries of the upper extremity may cause substantial loss of motor and sensory function resulting in alterations in both the peripheral and central nervous system (CNS) which may continue through recovery (Novak and Von Der Heyde, 2013 , 2015). Today, nerve transfer surgery plays a major role in nerve reconstruction, particularly in severe proximal nerve injuries (Tung and Mackinnon, 2010). Upon nerve transfer surgery (neurotization) an intact motor nerve from one muscle (donor nerve) is redirected to the distal undamaged portion of a nerve from another muscle (recipient nerve), effectively bypassing the injured segment of the nerve (Liu et al., 2012). Following nerve injury, timely reconstruction should be initiated since degeneration and fibrosis of motor end plates occurring within 1-2 years may preclude successful muscle re-innervation (Terzis and Papakonstantinou, 2000). Furthermore, in upper limb amputees, the concept of selective nerve transfers, known as targeted muscle re-innervation (TMR), has dramatically improved prosthetic arm and hand function (Kuiken et al., 2004, 2007; Dumanian et al., 2009).

It is well known that damage to peripheral nerves inevitably creates change at a central level, i.e., cortical reorganization which occurs following deafferentation of a respective area (Pons et al.,

Abbreviations: BP, brachial plexus; EMG, electromyography; sEMG, surface electromyography.
1991; Elbert et al., 1994; Flor, 2008). With increasing performance of nerve transfers and expanded clinical experience, experts in the field of nerve reconstruction have come to appreciate the important role of cortical plasticity and motor relearning during functional recovery following a nerve transfer (Anastakis et al., 2008). It has been shown that recovery after surgical nerve reconstruction is both a function of peripheral nerve regeneration and adaptations within the CNS, making use of the brain's plastic capacity (Dahlin et al., 2017).

As an example, intercostal-to-musculocutaneous nerve transfers are commonly used to re-innervate the biceps muscle in global brachial plexopathies (Millesi, 1977; Narakas, 1978; Terzis and Kostopoulos, 2007; Xiao et al., 2014). Upon successful regeneration of axons, motor control of the re-innervated biceps muscle initially requires activation of the intercostal nerves, i.e., through breathing and/or coughing (Carlstedt et al., 2004). Cognitive rehabilitation capitalizing on CNS plasticity allows patients to re-educate their brain and to gain volitional control of elbow flexion without activation of former intercostal nerve territories (Dahlin et al., 2017). Functional magnetic resonance imaging studies have shown that in patients with good biceps muscle re-innervation, induced and localized activity in the former biceps muscle cortical area is re-established, indicating cortical plasticity following successful nerve reconstruction (Malessy et al., 2003). Therefore, the importance of cortical changes and plasticity need not be underestimated during rehabilitation following motor nerve transfers (Novak, 2008).

Sensorimotor re-education following complex nerve reconstruction is a cognitively demanding process necessitating a structured neuro-rehabilitation program (Novak, 2008; Bergmeister et al., 2017). sEMG biofeedback has been widely used for rehabilitation of the upper extremity in stroke patients (Rayegani et al., 2014; Kim, 2017). In the nerve transfer patient, however, this biofeedback technique has not yet been described. Following nerve transfer surgery, the regeneration of motor axons requires a considerable period of time and patients will often struggle to attain control of volitional contractions in the re-innervated muscle (Kahn and Moore, 2016). Before visual or even palpable contractions occur sEMG can provide valuable feedback for the patient and guide rehabilitation focused on sensorimotor re-education. With the establishment of new motor patterns and cortical remapping, control of the re-innervated muscle will be attained without activation of the donor muscle after successful rehabilitation (Novak and Von Der Heyde, 2013).

Here, we introduce two rehabilitation protocols using surface EMG-guided biofeedback in different groups of nerve injury patients. The first group of patients includes patients with severe nerve injuries of the upper extremity undergoing nerve transfers 


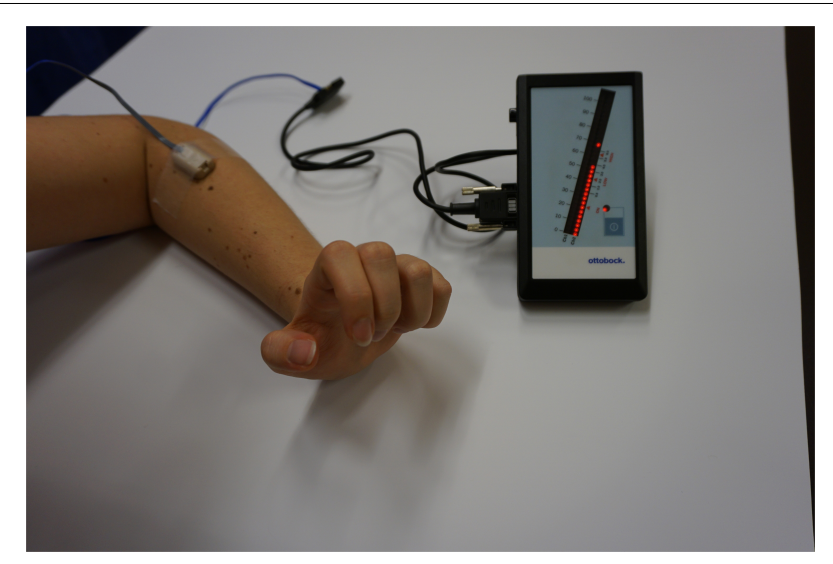

FIGURE 1 | Training with the MyoBoy (Ottobock, Duderstadt, Germany) with one dry electrode placed on the extensor compartment of the forearm. The EMG signal's amplitude is reflected by the LED dots. This set-up may be used for home training.

to restore biological arm and hand function. The second group includes patients in whom biological reconstruction has failed and extremity function was reconstructed with a myoelectric prosthesis.

\section{PROTOCOLS}

\section{Rehabilitation Protocol Using Surface EMG Biofeedback for Patients With Nerve Transfers to Restore Biological Upper Extremity Function}

Rehabilitation after nerve transfers is divided into three phases. In the first phase following surgery the nerves regenerate and no active motion is possible, referred to as "silent" phase (see Figure 3). This re-innervation process usually takes a considerable period of time. Therapy in this early stage, however, can be initiated for cortical activation by mirror therapy (see Figure $\mathbf{4 A}$ ), motor imagery and observation of movements (McCabe, 2011; Bowering et al., 2012). In mirror therapy, originally described to treat phantom limb pain by Ramachandran and Hirstein (1998), a patient places his normal hand on one side of a vertically placed mirror, which creates the illusion that the injured, amputated or denervated hand has returned and exhibits normal function. External electrical muscle stimulation may be of use to elicit movement of the paralyzed limb area, which also enhances cortical activation. This approach supports motor learning at a later stage. Additionally, therapy might also focus on body symmetry, trunk stability, and posture as well as preservation of range of motion for joints of the affected extremity.

The first volitional activation of the re-innervated muscle marks the start of the second phase of rehabilitation. Between 3 and 6 months post surgery we recommend monthly assessments of muscle activity using transcutaneous electrodes to identify

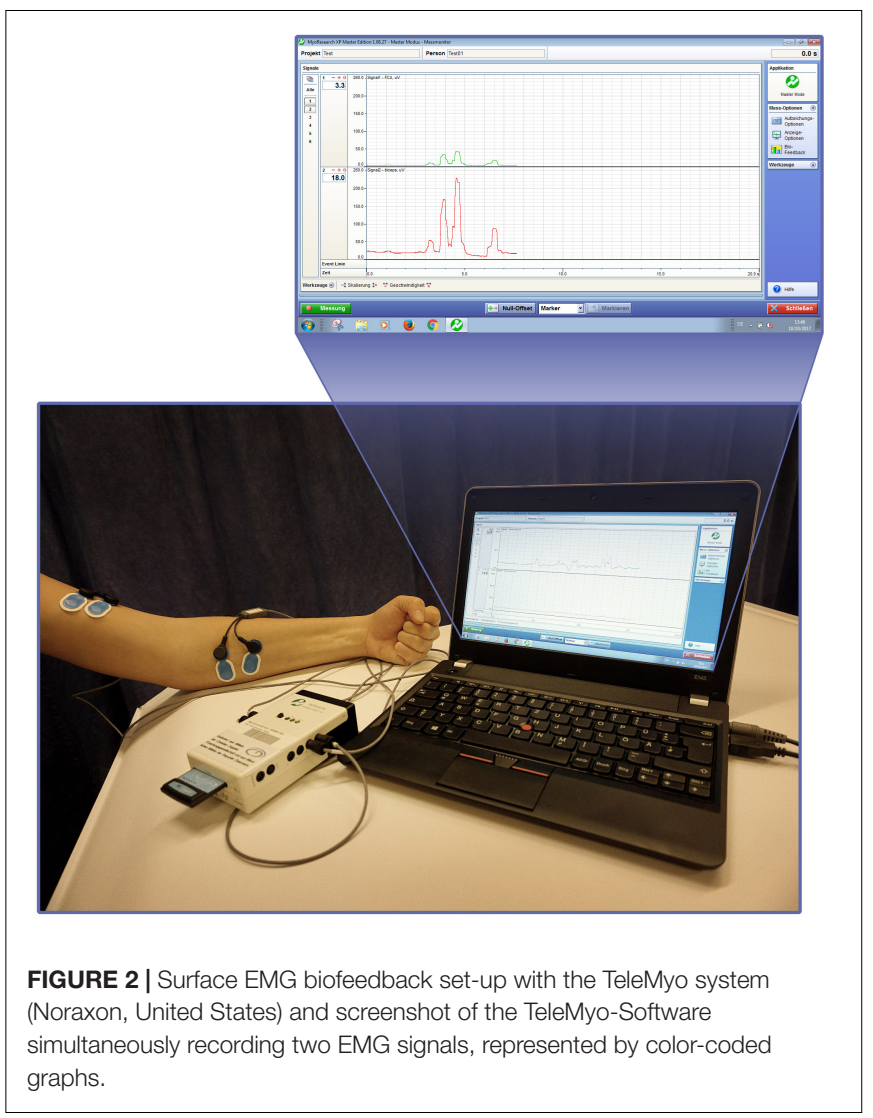

first volitional muscle activation. The initial re-innervation is confirmed, when the sEMG signal of the muscle activation repeatedly has an amplitude that is $2-3$ times higher than the amplitude during relaxation. This allows patient and therapist to see a distinct difference between muscle relaxation and activation. sEMG biofeedback training increases awareness of the new target muscle. Firstly, patients may not know how to activate a new target muscle. This is because activation requires initiation of movement patterns that the nerve had before its transfer (Novak and Von Der Heyde, 2015). For example, in case of Oberlin's ulnar nerve transfer, where a fascicular group of the ulnar nerve is transferred to the musculocutaneus nerve (Oberlin et al., 1994), the patient initially activates the biceps by thinking about "hand closing" or "activating the flexor carpi ulnaris (FCU)" (Oberlin et al., 2002). As this may be contra-intuitive for the patient without profound knowledge of the underlying anatomy, perioperative patient education is crucial. It ensures that they understand the consequence of nerve injury, the surgical procedure of the nerve transfer and the expected recovery (Novak and Von Der Heyde, 2013; Kahn and Moore, 2016).

By using sEMG biofeedback the therapist can identify individual, suitable movements for reliable muscular activation as an electrode is placed over the muscle of interest and the patient is asked to perform specific movements that the transferred nerve is originally responsible for (see Figure 4B). Additionally, sEMG is used to visualize muscle contraction during training, which is not visible or even palpable at 


\section{sEMG-guided rehabilitation}

\author{
Patients with nerve transfers to restore \\ biological upper extremity function

\section{Phase 1} \\ - axonal regeneration into target muscle \\ - „silent“ phase \\ - no active motion - patient education! \\ - early cortical activation of the formerly \\ deafferented area by mirror therapy, motor \\ imagery, observation of movements and external \\ electrical simulation \\ Phase 2 \\ - first volitional contractions \\ - guided sEMG training to facilitate initial \\ movements and to enhance cortical activity \\ - sEMG biofeedback increases awareness of the \\ new target muscle \\ Phase 3 \\ - relearning original movement patterns \\ - sEMG biofeedback to facilitate separation of \\ muscular activities \\ - refinement of motor skills marks end of \\ rehabilitation
}

Patients with bionic reconstruction

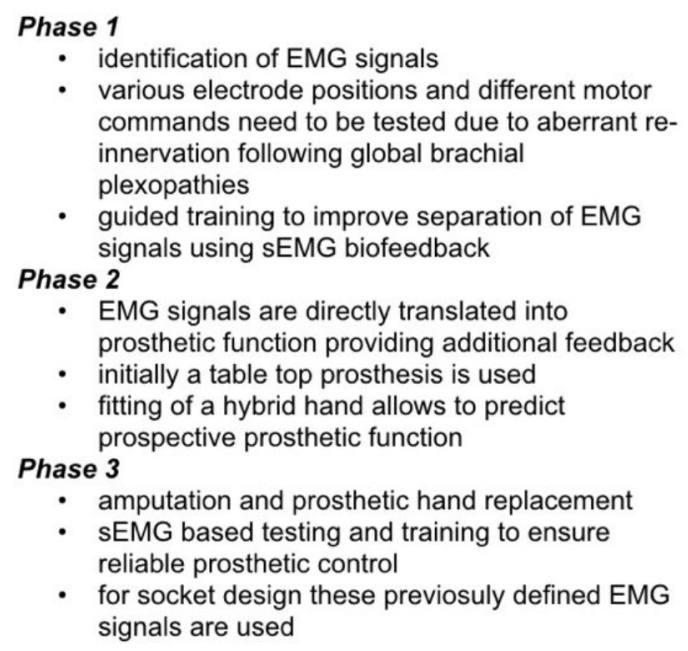

FIGURE 3 | Rehabilitation process of both patient groups.

that early stage of re-innervation. As soon as the patient knows how to activate the re-innervated muscle, he might think of a combination of the original muscle movement and the new activation pattern. In case of an Oberlin's ulnar nerve transfer this might include "elbow flexion" in combination with "hand closing" (see Figure 4C). As suggested by Novak, bilateral actions, i.e., performing the movement with both the injured and the healthy side, can be helpful for some patients (Novak, 2008; Novak and Von Der Heyde, 2013).

The third phase of rehabilitation starts as soon as the muscle strength is sufficient to overcome the inertia of the corresponding joint and initiate actual movement. Here, the focus lies on relearning the original movement pattern. After an Oberlin's nerve transfer this means flexion of the elbow without simultaneously closing the hand (see Figure 4D). The therapist encourages the patient to gradually activate the re-innervated muscle with decreasing activity of the supporting movements. Additionally, closing the hand without simultaneous contraction of the biceps muscle needs to be promoted through training. To support this cognitively demanding process, we encourage the use of sEMG biofeedback to attain reliable separation of muscle activity. Here, a setup with two EMG channels is recommended. One electrode is placed on the re-innervated muscle and the other on the original donor nerve muscle. This simultaneously visualizes the activity of both muscles. As shown in Figure 4D activation of one muscle without the other can be trained. The direct feedback using sEMG recordings provides the therapist as well as the patient with precise information about desirable and undesirable strategies for motor task execution. By the end of this third phase muscle force and fine motor skills should ideally meet the patient's as well as the clinician's expectations.

\section{Rehabilitation Protocol Using Surface EMG Biofeedback for Patients With Bionic Reconstruction}

The primary rehabilitation goal for patients eligible for bionic reconstruction is not to recover muscle strength. Instead, rehabilitation aims at establishing two independent EMG signals needed for reliable control of a myoelectric prosthesis after elective amputation (Salminger et al., 2016). The surgical concept and detailed treatment algorithm for bionic reconstruction can be found elsewhere (Aszmann et al., 2015; Hruby et al., 2017). In most global BP patients residual myoactivity may be detected in the fore- and upper arm, which - although without clinical significance - suffices to control a prosthetic hand. In these patients, sEMG training can be initiated without delay. In others, nerve and/or muscle transfers are needed to create additional EMG signals for future prosthetic control. sEMG training in these cases, therefore, starts with first volitional contractions of the new target muscles, approximately 6-9 months after nerve and/or muscle transfer. In this group, regular follow-ups where sEMG activity is assessed and documented, take place at 3, 6, and 9 months after surgery. In our experience nerve regeneration takes longer in this patient group and first volitional muscle activation is seldom detected before 6 months after surgery. As for patients with biological reconstruction of function, the initial re-innervation is confirmed, when the sEMG signal of the muscle activation repeatedly has an amplitude that is 2-3 time higher than the amplitude during relaxation. 


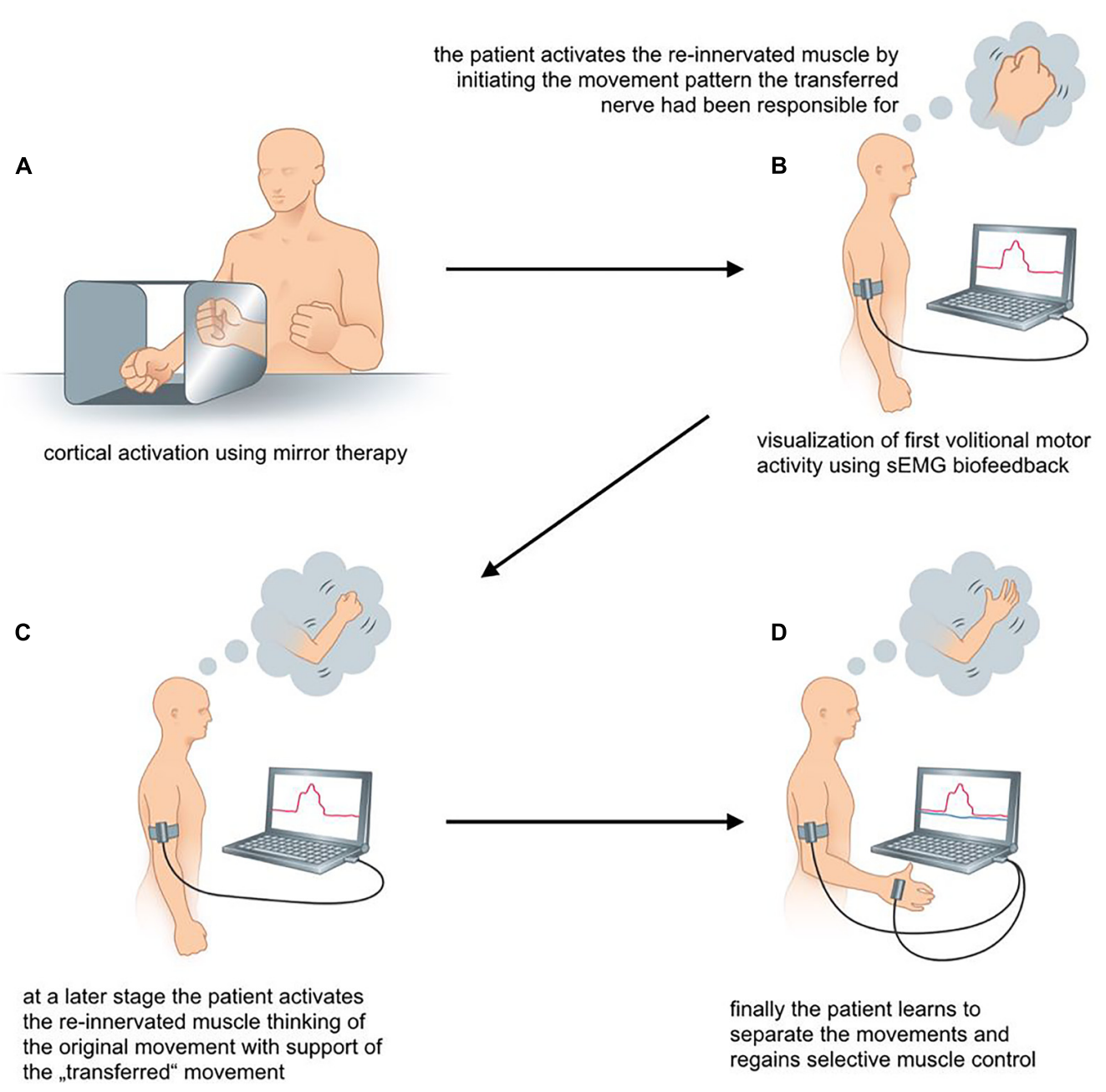

FIGURE 4 | (A-D) SEMG-guided rehabilitation for patients with biologic reconstruction of upper extremity function. The scheme illustrates the rehabilitation process following an Oberlin's ulnar nerve transfer.

The first phase of training includes the identification of EMG signals. The definition of the best positions for recording sEMG is critical and many possible electrode positions need to be compared by observing the amplitude of the EMG signal. Due to the aberrant re-innervation of muscles after global brachial plexopathies, the identification of movements that result in the greatest muscle activity, is usually complex. Cognitive motor commands might elicit movements which differ from biological patterns, e.g., the signal for closing the hand may be located at the dorsal aspect of the fore-arm. Therefore, even movements that seem illogical, or rather anatomically incorrect need to be tested. The advantage of sEMG in contrast to needle EMG arises from the possibility to adjust the electrode's position multiple times during testing, which is not feasible with needle EMG and might also cause pain. Additionally, the identification of sEMG signals is more relevant as later on transcutaneous electrodes within the prosthetic socket pick up these EMG signals and translate them into prosthetic hand function.
As soon as two different electrode locations with their unique activation pattern are established, sEMG training focuses on the separation of these signals (see Figure 5A). With the muscular activity visualized by the sEMG feedback device the patient tries to activate one muscle without the other. Also here, the direct feedback allows patient and therapist to try slight variations of the movements to find the best starting point for selective and consistent muscular activity.

During the second phase of rehabilitation the sEMG signal can be used for direct control of a table top prosthesis (as shown in Figure 5B). Although the prosthetic hand does not give as precise feedback as the visualization via EMG graphs, this approach allows to predict prosthetic hand function after amputation. Finally, patients are fitted with a hybrid hand, a fully functional prosthesis mounted on to or below the paralyzed hand. This gives a more realistic outlook on future prosthetic hand function, as illustrated in Figure 5C.

Elective amputation marks the beginning of the third phase of rehabilitation. sEMG based testing and training ensures that 
A

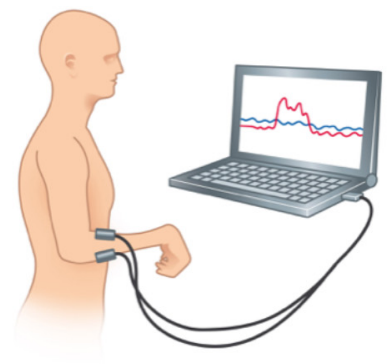

after identification of EMG signals their separation is trained using sEMG biofeedback

\section{C}

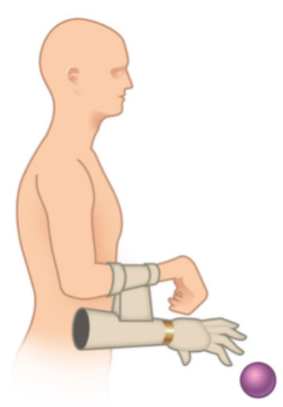

a hybrid prosthetic hand is fitted below the paralyzed hand to allow visualization of prospective prosthetic hand use
B

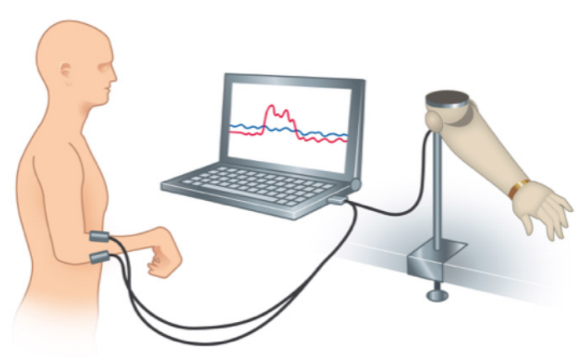

a table top prosthesis to experience and train possible prosthetic function

after amputation guided prosthetic training improves reliable prosthetic function

FIGURE 5 | (A-D) sEMG-guided rehabilitation for patients with bionic hand reconstruction.

the patient can still activate the two muscles independently. The signal positions can also be used for the design of the prosthetic socket, which is usually possible 4-6 weeks after amputation. As recommended in all amputees (Johnson and Mansfield, 2014; Resnik et al., 2014) regular prosthetic training (see Figure 5D) optimizes device control in activities of daily living and marks the end of rehabilitation.

\section{PATIENTS, METHODS, AND DESIGN OF FEASIBILITY STUDY}

We implemented the described protocols into clinics in eleven patients to test whether their application was feasible and help improve outcomes.

\section{Patients}

Patients who followed the described protocols had a severe injury of one or several peripheral nerves of the upper extremity that required a surgical reconstruction. Exclusion criteria were injuries of the CNS, untreated psychological disorders and unstable fractures of the upper extremity.

Depending on the injury and the intervention planned, patients were treated with either one of the rehabilitation protocols:
- Group 1: Patients with peripheral nerve injuries and selective nerve transfers to reconstruct biological upper limb function $(n=5)$.

- Group 2: Patients with severe peripheral nerve injuries, where biological reconstruction was deemed impossible. In these patients, prosthetic devices were used to restore hand function by technological means $(n=6)$. The concept of bionic reconstruction was recently described by Aszmann et al. (2015) and Hruby et al. (2017).

Patient characteristics can be found in Tables 1, 2. All patients received structured training with sEMG biofeedback. A summary of both rehabilitation approaches can be found in Figure 3 .

This clinical implementation was approved by the ethics committee of the Medical University of Vienna, Austria and carried out in accordance with the standards set by the Declaration of Helsinki. All patients provided written informed consent to participating in this study.

\section{Materials}

The EMG electrodes used in this study were bipolar and included a ground, circumventing the need of an extra ground electrode [product number: 13E202 $=50(50 \mathrm{~Hz})$, Ottobock Healthcare, Duderstadt, Germany]. 
TABLE 1 | Patient characteristics of Group 1, in whom biological restoration of upper limb function was performed.

\begin{tabular}{|c|c|c|c|c|}
\hline $\begin{array}{l}\text { Case } \\
\text { nr. }\end{array}$ & $\begin{array}{l}\text { Sex, age } \\
\text { (years) }\end{array}$ & $\begin{array}{l}\text { Type of } \\
\text { accident }\end{array}$ & Type of lesion & $\begin{array}{l}\text { Reconstructive surgeries for restoration } \\
\text { of upper limb function }\end{array}$ \\
\hline 1 & $\mathrm{~m}, 68$ & $\begin{array}{l}\text { Motorcycle } \\
\text { accident }\end{array}$ & $\begin{array}{l}\text { Polytrauma; global brachial } \\
\text { plexopathy }\end{array}$ & $\begin{array}{l}\text { Nerve grafts to bridge defect of MCN; thoracodorsal nerve grafts to bridge defect of } \\
\text { axillary nerve; nerve grafts for posterior trunk reconstruction; Oberlin's ulnar nerve } \\
\text { transfer to MCN motor branch to the short head of the biceps }\end{array}$ \\
\hline 2 & $m, 56$ & Bicycle accident & $\begin{array}{l}\text { Nerve root avulsion of } \\
\text { C5-C6 }\end{array}$ & $\begin{array}{l}\text { Oberlin's ulnar nerve transfer to MCN motor branch for restoration of biceps function; } \\
\text { transfer of radial triceps motor branch to axillary nerve }\end{array}$ \\
\hline 3 & $m, 62$ & Bicycle accident & $\begin{array}{l}\text { Extensive damage to } \\
\text { superior trunk of the } \mathrm{BP} \text {; } \\
\text { traction injury of } \mathrm{C} 7\end{array}$ & $\begin{array}{l}\text { XI-to-suprascapular nerve transfer; end-to-end transfer of phrenic nerve to } \mathrm{C} 7 \text {; transfer } \\
\text { of ulnar nerve fascicle to biceps motor branch of } \mathrm{MCN} \text {; transfer of median nerve fascicle } \\
\text { to brachialis motor branch of } \mathrm{MCN} \text {; transfer of radial nerve fascicle to axillary nerve }\end{array}$ \\
\hline 4 & $\mathrm{f}, 22$ & Car accident & $\begin{array}{l}\text { Nerve root avulsion of } \mathrm{C} 7 \\
\text { damage to } \mathrm{C} 8 \text { and } \mathrm{T} 1\end{array}$ & $\begin{array}{l}\text { Nerve grafts from } \mathrm{C} 5 \text { and } \mathrm{C} 6 \text { to } \mathrm{MCN} \text {, median and radial nerve; nerve grafts from C8 to } \\
\text { median, radial and ulnar nerve; nerve grafts from } \mathrm{T} 1 \text { to ulnar nerve }\end{array}$ \\
\hline 5 & $f, 43$ & $\begin{array}{l}\text { Minor trauma years } \\
\text { after OBPL }\end{array}$ & $\begin{array}{l}\text { Traction injury of superior } \\
\text { and medial trunk of the BP }\end{array}$ & $\begin{array}{l}\text { Nerve grafts to bridge defect of } \mathrm{C} 5, \mathrm{C} 6 \text {, and } \mathrm{C} 7 \text { to restore elbow function and shoulder } \\
\text { stability; transfer of median nerve fascicle to brachial motor branch of } \mathrm{MCN}\end{array}$ \\
\hline
\end{tabular}

BP, brachial plexus; MCN, musculocutaneous nerve; OBPL, obstetrical brachial plexus lesion; OP, operation; XI, spinal accessory nerve.

TABLE 2 | Patient characteristics of Group 2, in whom bionic reconstruction was initiated due to infeasibility of biological treatment alternatives.

\begin{tabular}{|c|c|c|c|c|}
\hline $\begin{array}{l}\text { Case } \\
\text { nr. }\end{array}$ & $\begin{array}{l}\text { Sex, age } \\
\text { (years) }\end{array}$ & $\begin{array}{l}\text { Type of } \\
\text { accident }\end{array}$ & Type of lesion & $\begin{array}{l}\text { Surgeries to improve biotechnological interface } \\
\text { after initial reconstructions have failed to } \\
\text { improve hand function }\end{array}$ \\
\hline 1 & m, 32 & Fall from height & $\begin{array}{l}\text { Avulsion of } \mathrm{C} 7-\mathrm{T} 1 \text {, traction injury of } \\
\text { the infraclavicular plexus }\end{array}$ & Elective amputation of the forearm \\
\hline 2 & $\mathrm{~m}, 32$ & Motorcycle accident & Rupture of all 3 trunci of the BP & $\begin{array}{l}\text { Free gracilis muscle transferred to forearm extensor compartment \& } \\
\text { neurotization of deep branch of radial nerve to obturator nerve; elective } \\
\text { amputation of the forearm }\end{array}$ \\
\hline 3 & $m, 55$ & Motorcycle accident & Avulsion of C5-T1 & Elective amputation of the upper arm \\
\hline 4 & $\mathrm{~m}, 38$ & Motorcycle accident & $\begin{array}{l}\text { Extensive damage to roots } \mathrm{C} 5-\mathrm{C} 8 \text {; } \\
\text { avulsion of } \mathrm{T} 1\end{array}$ & Elective amputation of the forearm \\
\hline 5 & $\mathrm{~m}, 27$ & Motorcycle accident & Avulsion C8-T1 & Elective amputation of the forearm \\
\hline 6 & m, 43 & Motorcycle accident & Avulsion of C6-T1 & $\begin{array}{l}\text { Transfer of triceps muscle to supraspinatus fossa and transfer of biceps } \\
\text { muscle to supraclavicular fossa to improve prosthetic fitting; elective } \\
\text { amputation of the arm (shoulder exarticulation) }\end{array}$ \\
\hline
\end{tabular}

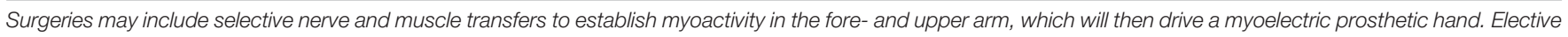

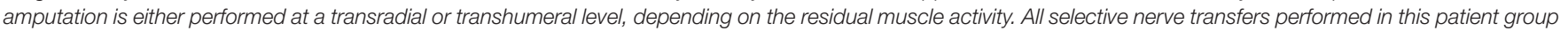
were successful.

All patients in group 2 used a SensorHand Speed@ (Ottobock Healthcare, Duderstadt, Germany) as their standard prosthetic device.

\section{Implementation of Rehabilitation Protocols}

In both groups the suggested procedures of the protocols could be implemented for all patients by one experienced therapist (AS). In group 1 differences in between subjects included the use of external electrical stimulation after nerve transfer surgery ( $n=3$ users, Cases 2, 3, and 4; $n=2$ non-users, Cases 1 and 5) using exponential current for denervated muscles and surge current for previously re-innervated muscles. In group 2 the myosignal identification for future prosthetic control was complicated by the mixed pattern of BP injury and the aberrant re-innervation that had occurred. Through the application of sEMG feedback, however, signals could be readily detected with multiple electrode positions and various motor commands guided by the therapist. The process of sEMG signal identification therefore lasted several hours as various, oftentimes counterintuitive motor commands needed to be tested in order to elicit contraction in the target muscle. For example, in Case 2 of group 2 aberrant re-innervation caused the signal for closing the hand to be located at the dorsal aspect of the fore-arm. Although the location of sEMG signals and corresponding motor commands to elicit contraction greatly varied inter-individually, we found that the majority of signals were located at the proximal third of the fore-arm (mostly pronator teres muscle, and extensor compartment). The time between nerve transfer surgery and first volitional muscle activation is outlined in Tables 3, 4 .

In both groups time in therapy depended on the patients' time limitations and the extent of injury. Individual adaptations were made with each patient. Guided training sessions using sEMG biofeedback with a therapist lasted $30 \mathrm{~min}$ to preclude muscle fatigue, which were usually offered once every 2 weeks for group 2. In group 1 time in therapy was intensified to once per week 
TABLE 3 | Upper limb function of patients with biologic reconstruction of hand function (patient group 1) before treatment and after end of therapy.

\begin{tabular}{|c|c|c|c|c|}
\hline Case nr. & $\begin{array}{l}\text { Upper limb function } \\
\text { including BMRC } \\
\text { grades at baseline }\end{array}$ & $\begin{array}{l}\text { Upper limb function } \\
\text { including BMRC } \\
\text { grades at follow-up }\end{array}$ & $\begin{array}{c}\text { Time between nerve transfer } \\
\text { surgery and first } \\
\text { volitional sEMG activity }\end{array}$ & $\begin{array}{l}\text { No. of therapy } \\
\text { sessions in total } \\
\text { (30 min each) }\end{array}$ \\
\hline \multirow[t]{5}{*}{1} & Deltoid muscle: 0 & Deltoid muscle: 2 & 5 months & 25 \\
\hline & Elbow flexion: 0 & Elbow flexion: 3 & & \\
\hline & Triceps muscle: 0 & Triceps muscle: 2 & & \\
\hline & No active hand & Wrist extension: 1 & & \\
\hline & function & Finger extension: 2 & & \\
\hline \multirow[t]{2}{*}{2} & Elbow flexion: 1 & Elbow flexion: 5 & 4 months & 22 \\
\hline & Deltoid muscle: 2- & Deltoid muscle: 5 & & \\
\hline \multirow[t]{5}{*}{3} & Elbow flexion: 0 & Elbow flexion: 5 & 3 months & 30 \\
\hline & Deltoid muscle: 0 & Deltoid muscle: 4 & & \\
\hline & Triceps muscle: 3 & Triceps muscle: 5 & & \\
\hline & Wrist extension: 3+ & Wrist extension: 5 & & \\
\hline & Finger flexion: $3+$ & Finger flexion: 5 & & \\
\hline \multirow[t]{3}{*}{4} & Elbow flexion: 0 & Elbow flexion: $3+$ & 5 months & 20 \\
\hline & Triceps muscle: 0 & Triceps muscle: 2 & & \\
\hline & No active hand function & $\begin{array}{l}\text { Wrist flexion: } 3 \\
\text { Finger flexion } \\
\text { (ulnar FDP part): } 3\end{array}$ & & \\
\hline \multirow[t]{3}{*}{5} & Elbow flexion: 0 & Elbow flexion: 3 & 4 months & 18 \\
\hline & Deltoid muscle: 2 & Deltoid muscle: 2 & & \\
\hline & Triceps muscle: $3+$ & Triceps muscle: 4 & & \\
\hline Mean ( \pm SD) & & & $4.2 \pm 0.75$ months & $23 \pm 4.20$ \\
\hline
\end{tabular}

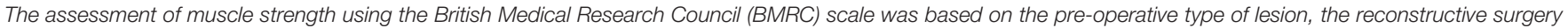

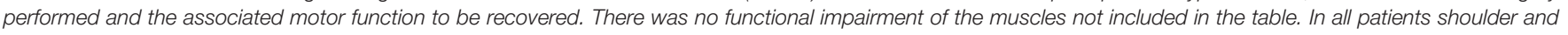

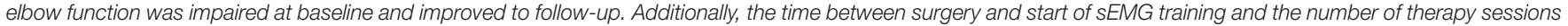
for each patient are presented.

during phase 2 to support motor re-education, whereas during phases 1 and 3 patients received therapy once a month.

The number of therapy sessions for each individual patient can be found in Tables 3, 4. All patients had the possibility to use EMG home training devices (see Figure 1), which was accepted by nine of eleven patients. The patients using the home training device all reported to have used it regularly and said it increased their training motivation due to intuitive feedback on muscle activity.

\section{Design of the Feasibility Study}

This was a within subjects pre- and post-test study. The baseline measurements of participants' upper limb function were performed after peripheral nerve injury and prior to surgical and therapeutical intervention. The follow-up measurements were conducted after the patients were discharged from rehabilitation.

\section{Functional Outcome Measures}

To evaluate hand and arm function, the British Medical Research Council (BMRC) (James, 2007) was used to assess muscle strength in patients with biological reconstruction (group 1). This grading system is the standard measure of muscle function after peripheral nerve injuries (Prosser and Conolly, 2005). In group 2 (bionic reconstruction) the ARAT (Action Research Arm test) was used to assess upper limb function (Lyle, 1981). This observational test consists of four sections with different tasks and a score maximum of 57 points (Lyle, 1981). It was performed before amputation (with the functionless "plexus" hand) as well as after final prosthetic fitting with the prosthetic hand.

\section{Statistics}

In accordance with the limited sample size of this study, in group 2 non-parametric tests were performed for the ARAT scores as these did not meet the requirement for normal distributions. Therefore, a paired 2-tailed Mann-Whitney $U$-test was used for the analysis. The significance level was set at Cronbach alpha $=0.05$. Explorative statistics were applied in group 1 for the BMRC grades. Statistical analysis was performed in SPSS 24 (IBM, Armonk, NY, United States).

\section{RESULTS}

Functional outcome measures for group 1 (biological reconstruction of upper limb function) are outlined in Table 3. Table 4 displays functional outcome measures for group 2 (bionic reconstruction with prosthetic hand replacement). All cases showed an improvement of hand function at the follow-up. The mean ARAT score improved significantly from $2.83 \pm 4.07$ to $25.00 \pm 10.94$ ( $p=0.028)$. In group 1 , shoulder and elbow function could be improved in all patients as measured by the BMRC scale. All patients regained an active elbow flexion against gravity (with scores obtained between M3 and M5). 
TABLE 4 | Scores of patients with bionic reconstruction (patient group 2) before treatment and after final prosthetic fitting.

\begin{tabular}{|c|c|c|c|c|}
\hline Case nr. & $\begin{array}{l}\text { ARAT at } \\
\text { baseline }\end{array}$ & $\begin{array}{l}\text { ARAT at } \\
\text { follow-up }\end{array}$ & $\begin{array}{l}\text { Start of sEMG } \\
\text { training }\end{array}$ & $\begin{array}{l}\text { No. of therapy } \\
\text { sessions in total } \\
\text { (30 min each) }\end{array}$ \\
\hline 1 & 7 & 35 & Immediately after first consultation & 24 \\
\hline 2 & 0 & 15 & $\begin{array}{l}\text { Training with one signal immediately after first consultation; second signal was } \\
\text { available } 9 \text { months after free gracilis muscle transfer + nerve transfer }\end{array}$ & 30 \\
\hline 3 & 0 & 19 & Immediately after first consultation & 16 \\
\hline 4 & 1 & 22 & Immediately after first consultation & 20 \\
\hline 5 & 9 & 42 & $\begin{array}{l}\text { Immediately after decision to aim for a bionic reconstruction as biologic } \\
\text { reconstruction failed }\end{array}$ & 20 \\
\hline 6 & 0 & 17 & Immediately after first consultation & 22 \\
\hline Mean $( \pm S D)$ & $2.83 \pm 4.07$ & $25.00 \pm 10.94$ & & $22 \pm 4.32$ \\
\hline
\end{tabular}

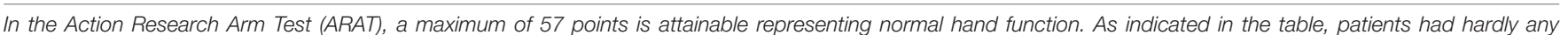

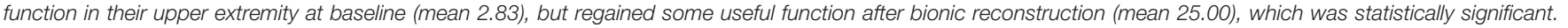
Additionally, the starting point for sEMG training and the number of therapy sessions for each patient are presented.

\section{DISCUSSION}

After peripheral nerve injury, immediate changes in the peripheral but also in the CNS occur, which continue through re-innervation and recovery (Novak and Von Der Heyde, 2015). Practice, repetition, and structured training programs with appropriate biofeedback are necessary to establish correct motor patterns (Novak and Von Der Heyde, 2013). Biofeedback using sEMG recordings has been shown to facilitate significant clinical improvements and to enhance the rehabilitation process in various neuromuscular diseases such as in stroke (Giggins et al., 2013; Huang et al., 2013; Oravitan and Avram, 2013; Neblett, 2016). In this paper we presented a structured rehabilitation protocol using sEMG biofeedback in patients with severe nerve injuries. Our clinical application included patients receiving nerve transfers to restore biological upper limb function as well as patients who underwent nerve surgeries to improve the future biotechnological interface, elective amputation and prosthetic hand replacement.

During the past decades, the use of nerve transfers has expanded with a wider range of applications and improved functional outcomes, particularly to restore biological extremity function in patients with severe proximal nerve injuries (Bertelli and Ghizoni, 2004; Novak, 2008; Bertelli and Ghizoni, 2010; Tung and Mackinnon, 2010; Mackinnon et al., 2012; Mackinnon, 2016). Still, waiting for a muscle function to recover is one of the greatest challenges for a patient after undergoing nerve transfer surgery. Especially in the early post-operative phase patients may be frustrated and/or depressed when no motor activity is seen (Kahn and Moore, 2016). This time period, where the patient feels that "nothing happens," is possibly shortened with the use of sEMG feedback as faint muscle activity is visualized before it is visible or even palpable. sEMG set-ups are valuable tools to localize those parts of a muscle with weak contractile actions, which would otherwise be unnoticed to the patient allowing an early start of training. Our patients reported that visualization of muscle activity before actual movements were possible helped them to stay motivated during rehabilitation. Additionally, the visualization of muscle activity increases awareness of the target muscle and facilitates a patient's understanding as to which motor command leads to the muscle activation.

As is in line with earlier studies (Tung et al., 2003; Bertelli and Ghizoni, 2011; Ray et al., 2011), patients in group 1 attained useful shoulder and upper arm function. In all five patients elbow function improved to a clinically relevant extent with active elbow flexion against gravity (M3) at follow-up. In two patients (Cases 2 and 3) where an Oberlin's ulnar nerve transfer had been performed, a score of M5 was obtained for elbow flexion. These results are better than those described by Bertelli and Ghizoni (2004) who used the same nerve transfer and obtained scores of M3 to M4. In a retrospective study by Ray et al. (2011) half of 29 patients obtained M4, eight scored M5, while the others had M3 or less, which is comparable to our results. Therefore, the results that were obtained after nerve transfer surgery were similar and in two cases slightly better than those reported in literature. While we believe that a structured rehabilitation protocol using sEMG biofeedback increases patient motivation and awareness, based on our current data we cannot conclude that clinical outcomes can be improved due to the small sample size and the fact that there was no control group. Additionally, it is well known that many factors influence the outcome of peripheral nerve surgery, such as patients' age and motivation (Novak and Von Der Heyde, 2013), the quality and concept of nerve reconstruction, type of lesion (Moran et al., 2005), etc. Therefore, also in future controlled studies it might be difficult to identify if sEMG can improve clinical outcomes.

For patients with global brachial plexopathies, in whom primary nerve reconstruction and secondary reconstructive procedures have failed to improve hand function, the concept of bionic reconstruction has proven successful to restore hand function via technological means (Aszmann et al., 2015). This novel treatment approach includes surgeries to improve the biotechnological interface, the elective amputation of the functionless hand and subsequent fitting with a mechatronic hand (Aszmann et al., 2015). In this patient population, the control of the prosthetic hand relies on the detection of voluntary residual muscle activity through EMG (Bergmeister et al., 2017). As muscle contraction in these patients will not 
result in biologically valuable function that is visible to the patient, biofeedback is considered an essential component of rehabilitation.

All patients in group 2 reported that they were highly satisfied with their decision to undergo bionic reconstruction and could reliably control their prosthesis after complementation of rehabilitation. The functional benefit could be confirmed by significant improvements in the ARAT (Action Research Arm Test) from $2.83 \pm 4.07$ to $25.00 \pm 10.94(p=0.028)$ on a scoring system from 0 to 57 . While this shows the great clinical improvement through bionic reconstruction and sEMG biofeedback training, it still needs to be noted that prosthetic reconstruction cannot fully restore human upper extremity function.

All eleven patients had the possibility to use EMG home training tools to further increase training time. Nine of them decided to take this possibility. The two patients who opted out reported that they did most of the home training protocol (muscle strengthening exercises) outside their home environment and therefore did not like to use an external device. Additionally, they felt that weekly training sessions with the therapist sufficed to improve motor function. Patients who did sEMG home training reported that using the MyoBoy (a simple, two channel EMG device, see Figure 1) made them feel more competent in controlling their EMG signals. As devices for sEMG visualization can be cheap and handy and were described as easy to operate, we strongly recommend their application to supplement therapy in the clinical environment.

Here, we introduced two structured rehabilitation protocols of sEMG-guided training in patients with nerve injuries. While clinical feasibility was proven in eleven patients undergoing structured rehabilitation, further research should include a controlled trial with a larger sample size to estimate the effect of the rehabilitation protocol on functional and psychosocial outcomes.

\section{REFERENCES}

Anastakis, D. J., Malessy, M. J., Chen, R., Davis, K. D., and Mikulis, D. (2008). Cortical plasticity following nerve transfer in the upper extremity. Hand Clin. 24, 425-444. doi: 10.1016/j.hcl.2008.04.005

Aszmann, O. C., Roche, A. D., Salminger, S., Paternostro-Sluga, T., Herceg, M., Sturma, A., et al. (2015). Bionic reconstruction to restore hand function after brachial plexus injury: a case series of three patients. Lancet 385, 2183-2189. doi: 10.1016/S0140-6736(14)61776-1

Bergmeister, K. D., Vujaklija, I., Muceli, S., Sturma, A., Hruby, L. A., Prahm, C., et al. (2017). Broadband prosthetic interfaces: combining nerve transfers and implantable multichannel EMG technology to decode spinal motor neuron activity. Front. Neurosci. 11:421. doi: 10.3389/fnins.2017. 00421

Bertelli, J. A., and Ghizoni, M. F. (2004). Reconstruction of C5 and C6 brachial plexus avulsion injury by multiple nerve transfers: spinal accessory to suprascapular, ulnar fascicles to biceps branch, and triceps long or lateral head branch to axillary nerve. J. Hand Surg. Am. 29, 131-139. doi: 10.1016/j.jhsa. 2003.10.013

Bertelli, J. A., and Ghizoni, M. F. (2010). Reconstruction of complete palsies of the adult brachial plexus by root grafting using long grafts and nerve transfers to target nerves. J. Hand Surg. Am. 35, 1640-1646. doi: 10.1016/j.jhsa.2010. 06.019

\section{CONCLUSION}

Successful neuromuscular rehabilitation requires detailed afferent feedback. Especially in the face of limb loss and/or BP lesions sEMG biofeedback may be used to bridge the time of recovery, where muscle contraction is otherwise unnoticed and help in the cognitively demanding process of establishing new motor patterns that eventually control the prosthetic replacement. After standard nerve transfer surgery individually tailored sEMG biofeedback can facilitate early sensorimotor reeducation, enhance patient motivation and compliance and thus improve clinical outcomes.

\section{AUTHOR CONTRIBUTIONS}

AS, LH, and OA conceived and designed the study. AS and LH performed the data acquisition. LH, AS, and CP analyzed the data. AS, LH, CP, JM, and OA interpreted the data, and wrote and edited the manuscript. All authors gave final approval for publication.

\section{FUNDING}

This study was funded by the Christian Doppler Research Foundation of the Austrian Council for Research and Technology Development and the Austrian Federal Ministry of Science, Research and Economy.

\section{ACKNOWLEDGMENTS}

We are grateful to Aron Cserveny for preparation of the illustrations included in the manuscript.

Bertelli, J. A., and Ghizoni, M. F. (2011). Results and current approach for brachial plexus reconstruction. J. Brachial Plex. Peripher. Nerve Inj. 6:2. doi: 10.1186/ 1749-7221-6-2

Bowering, K. J., O’Connell, N. E., Tabor, A., Catley, M. J., Leake, H. B., Moseley, G. I., et al. (2012). The effects of graded motor imagery and its components on chronic pain: a systematic review and meta-analysis. J. Pain 14, 3-13. doi: 10.1016/j.jpain.2012.09.007

Carlstedt, T., Anand, P., Htut, M., Misra, P., and Svensson, M. (2004). Restoration of hand function and so called "breathing arm" after intraspinal repair of C5-T1 brachial plexus avulsion injury. Case report. Neurosurg. Focus 16:E7. doi: $10.3171 /$ foc. 2004.16 .5 .8

Cram, J. R. (2003). The history of surface electromyography. Appl. Psychophysiol. Biofeedback 28, 81-91. doi: 10.1023/A:1023802407132

Dahlin, L. B., Andersson, G., Backman, C., Svensson, H., and Bjorkman, A. (2017). Rehabilitation, using guided cerebral plasticity, of a brachial plexus injury treated with intercostal and phrenic nerve transfers. Front. Neurol. 8:72. doi: 10.3389/fneur.2017.00072

Dumanian, G. A., Ko, J. H., O’shaughnessy, K. D., Kim, P. S., Wilson, C. J., and Kuiken, T. A. (2009). Targeted reinnervation for transhumeral amputees: current surgical technique and update on results. Plast. Reconstr. Surg. 124, 863-869. doi: 10.1097/PRS.0b013e3181b038c9

Elbert, T., Flor, H., Birbaumer, N., Knecht, S., Hampson, S., Larbig, W., et al. (1994). Extensive reorganization of the somatosensory cortex in adult humans 
after nervous system injury. Neuroreport 5, 2593-2597. doi: 10.1097/00001756199412000-00047

Flor, H. (2008). Maladaptive plasticity, memory for pain and phantom limb pain: review and suggestions for new therapies. Expert Rev. Neurother. 8, 809-818. doi: 10.1586/14737175.8.5.809

Giggins, O. M., Persson, U. M., and Caulfield, B. (2013). Biofeedback in rehabilitation. J. Neuroeng. Rehabil. 10:60. doi: 10.1186/1743-0003$10-60$

Hruby, L. A., Sturma, A., Mayer, J. A., Pittermann, A., Salminger, S., and Aszmann, O. C. (2017). Algorithm for bionic hand reconstruction in patients with global brachial plexopathies. J. Neurosurg. 127, 1163-1171. doi: 10.3171/2016. 6.JNS16154

Huang, H., Lin, J. J., Guo, Y. I., Wang, W. T. J., and Chen, Y. J. (2013). EMG biofeedback effectiveness to alter muscle activity pattern and scapular kinematics in subjects with and without shoulder impingement. J. Electromyogr. Kinesiol. 23, 267-274. doi: 10.1016/j.jelekin.2012.09.007

James, M. A. (2007). Use of the medical research council muscle strength grading system in the upper extremity. J. Hand Surg. Am. 32, 154-156. doi: 10.1016/j. jhsa.2006.11.008

Johnson, S. S., and Mansfield, E. (2014). Prosthetic training: upper limb. Phys. Med. Rehabil. Clin. N. Am. 25, 133-151. doi: 10.1016/j.pmr.2013. 09.012

Kahn, L. C., and Moore, A. M. (2016). Donor activation focused rehabilitation approach: maximizing outcomes after nerve transfers. Hand Clin. 32, 263-277. doi: $10.1016 /$ j.hcl.2015.12.014

Kim, J. H. (2017). The effects of training using EMG biofeedback on stroke patients upper extremity functions. J. Phys. Ther. Sci. 29, 1085-1088. doi: 10.1589/jpts. 29.1085

Kuiken, T. A., Dumanian, G. A., Lipschutz, R. D., Miller, L. A., and Stubblefield, K. A. (2004). The use of targeted muscle reinnervation for improved myoelectric prosthesis control in a bilateral shoulder disarticulation amputee. Prosthet. Orthot. Int. 28, 245-253.

Kuiken, T. A., Miller, L. A., Lipschutz, R. D., Lock, B. A., Stubblefield, K., Marasco, P. D., et al. (2007). Targeted reinnervation for enhanced prosthetic arm function in a woman with a proximal amputation: a case study. Lancet 369, 371-380. doi: 10.1016/S0140-6736(07)60193-7

Liu, Y., Lao, J., Gao, K., Gu, Y., and Xin, Z. (2012). Outcome of nerve transfers for traumatic complete brachial plexus avulsion: results of 28 patients by DASH and NRS questionnaires. J. Hand Surg. Eur. Vol. 37, 413-421. doi: 10.1177/ 1753193411425330

Lyle, R. C. (1981). A performance test for assessment of upper limb function in physical rehabilitation treatment and research. Int. J. Rehabil. Res. 4, 483-492. doi: 10.1097/00004356-198112000-00001

Mackinnon, S. E. (2016). Donor distal, recipient proximal and other personal perspectives on nerve transfers. Hand Clin. 32, 141-151. doi: 10.1016/j.hcl.2015. 12.003

Mackinnon, S. E., Yee, A., and Ray, W. Z. (2012). Nerve transfers for the restoration of hand function after spinal cord injury. J. Neurosurg. 117, 176-185. doi: 10.3171/2012.3.JNS12328

Malessy, M. J., Bakker, D., Dekker, A. J., Van Duk, J. G., and Thomeer, R. T. (2003). Functional magnetic resonance imaging and control over the biceps muscle after intercostal-musculocutaneous nerve transfer. J. Neurosurg. 98, 261-268. doi: $10.3171 /$ jns.2003.98.2.0261

McCabe, C. (2011). Mirror visual feedback therapy. A practical approach. J. Hand Ther. 24, 170-178. doi: 10.1016/j.jht.2010.08.003

Millesi, H. (1977). Surgical management of brachial plexus injuries. J. Hand Surg. Am. 2, 367-378. doi: 10.1016/S0363-5023(77)80046-4

Moran, S. L., Steinmann, S. P., and Shin, A. Y. (2005). Adult brachial plexus injuries: mechanism, patterns of injury, and physical diagnosis. Hand Clin. 21, 13-24. doi: 10.1016/j.hcl.2004.09.004

Narakas, A. (1978). Surgical treatment of traction injuries of the brachial plexus. Clin. Orthop. Relat. Res. 133, 71-90.

Neblett, R. (2016). Surface electromyographic (SEMG) biofeedback for chronic low back pain. Healthcare 4:E27. doi: 10.3390/healthcare4020027

Novak, C. B. (2008). Rehabilitation following motor nerve transfers. Hand Clin. 24, 417-423. doi: 10.1016/j.hcl.2008.06.001
Novak, C. B., and Von Der Heyde, R. L. (2013). Evidence and techniques in rehabilitation following nerve injuries. Hand Clin. 29, 383-392. doi: 10.1016/ j.hcl.2013.04.012

Novak, C. B., and Von Der Heyde, R. L. (2015). Rehabilitation of the upper extremity following nerve and tendon reconstruction: when and how. Semin. Plast. Surg. 29, 73-80. doi: 10.1055/s-0035-1544172

Oberlin, C., Ameur, N. E., Teboul, F., Beaulieu, J. Y., and Vacher, C. (2002). Restoration of elbow flexion in brachial plexus injury by transfer of ulnar nerve fascicles to the nerve to the biceps muscle. Tech. Hand Up. Extrem. Surg. 6, 86-90. doi: 10.1097/00130911-200206000-00007

Oberlin, C., Beal, D., Leechavengvongs, S., Salon, A., Dauge, M. C., and Sarcy, J. J. (1994). Nerve transfer to biceps muscle using a part of ulnar nerve for C5C6 avulsion of the brachial plexus: anatomical study and report of four cases. J. Hand Surg. Am. 19, 232-237. doi: 10.1016/0363-5023(94)90011-6

Oravitan, M., and Avram, C. (2013). The effectiveness of electromyographic biofeedback as part of a meniscal repair rehabilitation programme. J. Sports Sci. Med. 12, 526-532.

Pons, T. P., Garraghty, P. E., Ommaya, A. K., Kaas, J. H., Taub, E., and Mishkin, M. (1991). Massive cortical reorganization after sensory deafferentation in adult macaques. Science 252, 1857-1860. doi: 10.1126/science.1843843

Prosser, R., and Conolly, W. B. (2005). Rehabilitation of the Hand \& Upper Extremity. Amsterdam: Elsevier Limited.

Ramachandran, V. S., and Hirstein, W. (1998). The perception of phantom limbs. The D. O. hebb lecture. Brain 121(Pt 9), 1603-1630.

Ray, W. Z., Pet, M. A., Yee, A., and Mackinnon, S. E. (2011). Double fascicular nerve transfer to the biceps and brachialis muscles after brachial plexus injury: clinical outcomes in a series of 29 cases. J. Neurosurg. 114, 1520-1528. doi: 10.3171/2011.1.JNS10810

Rayegani, S. M., Raeissadat, S. A., Sedighipour, L., Rezazadeh, I. M., Bahrami, M. H., Eliaspour, D., et al. (2014). Effect of neurofeedback and electromyographic-biofeedback therapy on improving hand function in stroke patients. Top Stroke Rehabil. 21, 137-151. doi: 10.1310/tsr2102-137

Resnik, L., Klinger, S. L., Korp, K., and Walters, L. S. (2014). Training protocol for powered shoulder prosthesis. J. Rehabil. Res. Dev. 51, vii-xvi. doi: 10.1682/ JRRD.2014.07.0162

Salminger, S., Roche, A. D., Hruby, L. A., Sturma, A., Riedl, O., Bergmeister, K. D., et al. (2016). Prosthetic reconstruction to restore function in transcarpal amputees. J. Plast. Reconstr. Aesthet. Surg. 69, 305-310. doi: 10.1016/j.bjps.2015. 10.029

Terzis, J. K., and Kostopoulos, V. K. (2007). The surgical treatment of brachial plexus injuries in adults. Plast. Reconstr. Surg. 119, 73e-92e. doi: 10.1097/01. prs.0000254859.51903.97

Terzis, J. K., and Papakonstantinou, K. C. (2000). The surgical treatment of brachial plexus injuries in adults. Plast. Reconstr. Surg. 106, 1097-1122. doi: 10.1097/ 00006534-200010000-00022

Tung, T. H., and Mackinnon, S. E. (2010). Nerve transfers: indications, techniques, and outcomes. J. Hand Surg. 35, 332-341. doi: 10.1016/j.jhsa.2009.12.002

Tung, T. H., Novak, C. B., and Mackinnon, S. E. (2003). Nerve transfers to the biceps and brachialis branches to improve elbow flexion strength after brachial plexus injuries. J. Neurosurg. 98, 313-318. doi: 10.3171/jns.2003.98.2.0313

Xiao, C., Lao, J., Wang, T., Zhao, X., Liu, J., and Gu, Y. (2014). Intercostal nerve transfer to neurotize the musculocutaneous nerve after traumatic brachial plexus avulsion: a comparison of two, three, and four nerve transfers. J. Reconstr. Microsurg. 30, 297-304. doi: 10.1055/s-0033-1361840

Conflict of Interest Statement: The authors declare that the research was conducted in the absence of any commercial or financial relationships that could be construed as a potential conflict of interest.

Copyright (C) 2018 Sturma, Hruby, Prahm, Mayer and Aszmann. This is an openaccess article distributed under the terms of the Creative Commons Attribution License (CC BY). The use, distribution or reproduction in other forums is permitted, provided the original author(s) and the copyright owner(s) are credited and that the original publication in this journal is cited, in accordance with accepted academic practice. No use, distribution or reproduction is permitted which does not comply with these terms. 